\title{
Mercado de madeiras tropicais: substituição na demanda de exportação
}

\author{
Alexandre Nascimento de ALMEIDA', Humberto ANGELO², João Carlos Garzel Leodoro da SILVA³, Vitor \\ Afonso HOEFLICH ${ }^{4}$ \\ RESUMO \\ Neste trabalho foi abordado o grau de substituição de seis espécies da Amazônia no mercado internacional de madeira serrada. \\ Utilizou-se como base metodológica o modelo de elasticidade de substituição. Os dados usados no modelo são mensais e \\ foram coletados na Secretaria de Comércio Exterior do Brasil (SECEX) para o período de janeiro de 1996 a setembro de \\ 2007. As espécies analisadas foram: mogno (Swietenia macrophylla), cedro (Cedrela spp.), virola (Virola surinamensis), louro \\ (Nectandra spp. e Ocotea spp.), angico (Anadenanthera spp.) e ipê (Tabebuia spp.). As elasticidades estimadas indicaram que \\ todas as espécies são boas substitutas ao mogno. Para as espécies que possuem características físicas diferentes, pode-se inferir \\ que fatores de mercado relacionados à garantia de fornecimento do mogno influenciaram os resultados encontrados. Em \\ geral, os resultados sugeriram uma semelhança entre as espécies consideradas nobres (mogno, ipê e cedro) para o mercado \\ internacional, indicando-as como boas substitutas entre si.
}

PALAVRAS-CHAVE: madeira tropical, elasticidade de substituição, mercado internacional.

\section{Tropical sawnwood market: substitution export demand}

\section{ABSTRACT}

This work analyzed the level of substitution of six Amazonian species on the international sawnwood market, by employing the substitution elasticity model. Data supplied by the Brazilian Department of Foreign Trade (SECEX) was collected monthly from January/1996 to September/2007. The species analyzed were: mogno (Swietenia macrophylla), cedro (Cedrela spp.), virola (Virola surinamensis), louro (Nectandra spp. e Ocotea spp.), angico (Anadenanthera spp.) e ipê (Tabebuia spp.). The elasticities indicated that all of the species as substitutes for mogno. For the species which possess different physical characteristics, market factors probably related to the supply guarantee of mogno have influenced these results. In general, the results suggested a similarity among the species considered noble (mogno, ipê and cedro) on the international market indicating them as good substitutes among themselves.

KEYWORDS: tropical sawnwood, substitution elasticity, international market.

\footnotetext{
1 Universidade Federal do Paraná - UFPR - alex.floresta@hotmail.com

2 Universidade de Brasília - UnB - humb@unb.br

${ }^{3}$ Universidade Federal do Paraná - UFPR - garzel@ufpr.br

${ }^{4}$ Universidade Federal do Paraná - UFPR - hoefich@ufpr.br
} 


\section{INTRODUÇÃO}

Neste trabalho é abordada a demanda de exportação de madeiras tropicais. Em especial, busca estimar o grau de substituição entre as principais espécies da Amazônia no mercado internacional de madeiras tropicais.

A atividade madeireira é uma das alternativas para o desenvolvimento da Amazônia. Atualmente, cerca de 5\% da populaçáo economicamente ativa da chamada Amazônia Legal (território que inclui todos os Estados da regiáo Norte, além do Mato Grosso e parte do Estado do Maranháo) trabalha direta ou indiretamente com a atividade madeireira (Lentini et al., 2003).

O País produz cerca de 25 milhóes de metros cúbicos de madeiras tropicais em toras e consome 13 milhôes $\mathrm{m}^{3}$ de madeira serrada, colocando-se, portanto, na liderança mundial de produção e consumo no setor. No entanto, como exportador o Brasil ocupa a quarta posição entre os países exportadores, com um montante comercializado inferior a 2 milhôes de $\mathrm{m}^{3}$ de madeira serrada em 2007 (ITTO, 2007).

A indústria de madeiras tropicais no Brasil tem sido estudada quanto à estrutura, à produçáo e ao mercado de seus produtos por vários autores; entre estes, destacam-se os trabalhos de Mercado (1980), Queiroz (1983), Santos (1986), Santos e Hummel (1988), Angelo e Silva (1997), Angelo (1998), Brasil (2003), Calderon e Angelo (2006) e outros.

Entretanto, para o Brasil se tornar um grande player no mercado internacional de madeira tropical, deve ser garantida uma produção madeireira estável e sustentável a longo prazo, respeitando-se as crescentes exigências ambientais, muitas ainda a serem observadas, e proporcionando benefícios sociais à populaçáo da região.

Estudos indicaram que a maioria da produção madeireira da regiâo amazônica é considerada predatória ou oriunda de desmatamento e que, da produção dita sustentável, grande parte advinha de planos de manejo deficientes (Barreto et al., 2002). Segundo esses autores, a falta de pessoal treinado e a não utilização de equipamentos adequados resultam em danos excessivamente altos à floresta.

Algumas dificuldades enfrentadas para a efetiva implantação de um manejo florestal sustentável estão relacionadas à necessidade de alto investimento inicial, à burocracia dos órgãos reguladores, à concorrência desleal com madeireiras clandestinas e à falta de segurança fundiária na regiâo amazônica. Sabogal et al. (2005) destacaram como fatores econômicos limitantes à adoção do manejo: a) o baixo preço da madeira (legal) no mercado; b) a dificuldade de inserir novas espécies no mercado externo; e c) a falta de pesquisa sobre novas espécies.

Há grande esforço de entidades de pesquisa em analisar as características físicas de espécies desconhecidas do mercado ou de baixo valor comercial, objetivando encontrar espécies substitutas às de maior valor comercial. Sem prejuízo de outras instituiçôes, pode-se citar o Instituto Brasileiro do Meio Ambiente e Recursos Renováveis (IBAMA), por meio do Laboratório de Produtos Florestais (LPF).

Entretanto, poucos estudos analisaram a influência de fatores de mercado na diferenciação das espécies, como: preço, qualidade, garantia de fornecimento, costumes, arranjos políticos, institucionais, creditícios, entre outros (Fontes e Barbosa, 1991). Assim, uma caracterização física semelhante não pressupôe, necessariamente, uma substituição das espécies no mercado.

Tendo em vista os fatos citados anteriormente, tornase importante aprofundar os estudos econômicos para as espécies amazônicas. Assim, o objetivo deste trabalho foi estimar o grau de substituição entre as principais espécies da Amazônia no mercado internacional de madeira serrada. Com isso, objetivou-se aqui fornecer informaçôes relevantes para a adoção de estratégias para apoiar o incremento das exportaçôes de madeira tropical, por meio do entendimento do grau de substituição existente entre espécies brasileiras no mercado internacional.

Dois aspectos merecem destaque acerca da importância do mercado internacional no desenvolvimento do manejo florestal sustentável. Primeiramente, o preço da madeira no mercado externo é muito maior que no Brasil: enquanto o metro cúbico de ipê foi comercializado a $\mathrm{R} \$ 1.200,00$ no mercado interno, no exterior era vendido por $\mathrm{R} \$ 2.200$ (PRÓ EXPORT, 2003). Em segundo lugar, há o efeito das exportaçóes no aumento do preço da madeira no mercado interno (Ângelo, 2006).

\section{MATERIAL E MÉTODOS}

Os dados utilizados neste trabalho compreenderam séries mensais de madeira serrada (espessura $>6 \mathrm{~mm}$ ) entre o período de jan/1996 e set/2007 (141 observaçôes). As séries foram coletadas na Secretaria de Comércio Exterior (SECEX, 2007), por meio do Sistema de Análise das Informaçóes de Comércio Exterior via Internet (ALICE-Web).

Foram utilizadas séries referentes à quantidade (volume) e preço exportado de seis espécies provenientes da Amazônia. A evoluçáo do preço foi obtida através da razáo entre o valor e a quantidade exportada. A série de preço foi corrigida através do Índice de Preço ao Consumidor dos Estados Unidos (CPI), obtida no Instituto de Pesquisa Econômica Aplicada (IPEA, 2008).

As espécies analisadas foram mogno (Swietenia macrophylla), cedro (Cedrela spp.), virola (Virola surinamensis), louro (Nectandra spp. e Ocotea spp.), angico (Anadenanthera spp.) e ipê (Tabebuia spp.). O pequeno número de espécies analisadas 
foi devido à disponibilidade limitada de dados desagregados no período recente.

Aplicou-se o método da Elasticidade de Substituição (ES), um dos conceitos mais conhecidos da teoria econômica e que foi introduzido (referenciado) no início da década de 1930 por Hicks $(1932)^{1}$ e Robinson $(1933)^{2}$, citados por Lima (2000).

Conforme Medeiros e Teixeira (1996), é comum o uso de modelos de ES em estudos que pressupóem diferenciação de produtos na agricultura. No setor florestal, destacam-se os trabalhos de Brasil (2002) e Ângelo, Prado e Brasil (2004).

Brasil (2002) utilizou a ES na estimativa do grau de substituição entre os painéis exportados pelo Brasil e entre os principais países exportadores, enquanto Ângelo, Prado e Brasil (2004) aplicaram a ES para verificar a substituição entre a madeira tropical de manejo e o desmatamento no mercado doméstico.

A fundamentação teórica microeconômica da elasticidade de substituição baseia-se na seguinte relação (equação 1):

$\varepsilon=\frac{d\left(q_{1} / q_{2}\right)}{d\left(d q_{2} / d q_{1}\right)} * \frac{d q_{2} / d q_{1}}{q_{1} / q_{2}}=\frac{d \log \left(q_{1} / q_{2}\right)}{d \log \left(d q_{2} / d q_{1}\right)}$

Conforme a equação 1 , a ES entre dois produtos $(\varepsilon)$ é medida pela taxa de variação percentual nas quantidades relativas de $q_{1} / q_{2}$, em razão da variação percentual na taxa marginal de substituição de $q_{2}$ por $q_{1}$.

Assim, dada a maximização da utilidade com restrição orçamentária, $d q_{2} / d q_{1}=p_{1} / p_{2}$, encontra-se a seguinte definição empírica de elasticidade de substituição:

$\varepsilon=\frac{d\left(q_{1} / q_{2}\right)}{d\left(p_{1} / p_{2}\right)} * \frac{p_{1} / p_{2}}{q_{1} / q_{2}}=\frac{d \log \left(q_{1} / q_{2}\right)}{d \log \left(p_{1} / p_{2}\right)}$

Essa definição pode ser apresentada na seguinte forma de equação:

$\log \left(q_{1} / q_{2}\right)=a+\varepsilon \log \left(p_{1} / p_{2}\right)_{(3)}$

e estimada estatisticamente por:

$$
\log \left(q_{1} / q_{2}\right)=a+\varepsilon \log \left(p_{1} / p_{2}\right)_{(4)}
$$

em que:

$q_{1}=$ quantidade da espécie 1 ofertada no momento $t$ para um mercado $x$;

$q_{2}=$ quantidade da espécie 2 ofertada no momento $t$ para um mercado $x$;

$\varepsilon=$ elasticidade de substituição;

1 HICKS, J.R. 1932. The theory of wages. Londres: MacMillan.

2 ROBINSON, J. 1933. The economics of imperfect competition. Londres: MacMillan. $p_{1}=$ preço da espécie 1 ofertada no momento $t$ para um mercado $x$;

$p_{2}=$ preço da espécie 2 ofertada no momento $t$ para um mercado $x$; e

$$
\beta=\text { termo estocástico. }
$$

As hipóteses testadas são $\mathrm{H}_{0}: \varepsilon=0$ e $\mathrm{H}_{1}: \varepsilon \square \neq 0$; em outros termos, se estatisticamente existe relação de substituição entre duas espécies tropicais de madeira serrada no mercado internacional. Assim, a redução no preço internacional de uma espécie provoca aumento de sua exportação e queda nas exportações das espécies substitutas, ceteris paribus, e vice-versa. Os resultados são entendidos em módulo; os valores entre 0 e 1 configuram relações inelásticas, e aqueles acima de 1 , elásticas.

A estimativa da ES (equação 4) foi realizada por meio do método dos Mínimos Quadrados Ordinários (MQO), e as hipóteses foram testadas através do teste $t$. Foram admitidas elasticidades significativas com uma probabilidade de erro de até $10 \%$ de probabilidade.

\section{RESULTADOS E DISCUSSÃO}

Os resultados e respectivos níveis de significância para as elasticidades estimadas foram apresentados na Tabela 1.

Todas as espécies analisadas se apresentaram como boas substitutas ao mogno, insinuando uma baixa diferenciação entre elas para o mercado externo. Essas estimativas mostram que, para um aumento de $1 \%$ nas relaçóes de preço entre o mogno/cedro, mogno/virola, mogno/louro, mogno/angico e

Tabela 1 - Estimativa da Elasticidade de Substituição entre seis espécies tropicais no mercado internacional de madeira serrada

\begin{tabular}{lccccc}
\hline Espécie & mogno & cedro & virola & louro & angico \\
\hline mogno & & & & & \\
cedro & $-2,2^{\star}$ & & & & \\
virola & $-1,4^{*}$ & ns & & & \\
louro & $-1,5^{\star}$ & ns & ns & & \\
angico & $-0,6^{\star *}$ & ns & ns & $-1,3^{*}$ & \\
ipê & $-1,2^{\star \star *}$ & $2,4^{*}$ & ns & ns & ns \\
\hline
\end{tabular}

Fonte: Elaborado pelos autores.

Nota: ns (não significativo), * (significativo a 1\% de probabilidade), ** (significativo a $5 \%$ de probabilidade) $e^{* \star \star}$ (significativo a $10 \%$ de probabilidade).

mogno/ipê, espera-se uma queda de 2,$2 ; 1,4 ; 1,5 ; 0,6$; e 1,2\% nas exportaçôes de mogno em relação a cedro, virola, louro, angico e ipê, respectivamente.

Além do alto grau de substituição entre o cedro e o mogno $(-2,2)$, esta relação se apresentou como uma boa substituta ao ipê $(2,4)$. Outra relação encontrada foi a do louro e angico, que mostraram grau de substituição de 1,3\%. 
Os resultados indicaram o mogno com um grande número de espécies substitutas comparativamente às demais madeiras analisadas, indicando a dificuldade de explicar as elasticidades encontradas apenas pelas características físicas das espécies.

Especificamente em relação ao mogno, uma espécie de madeira nobre, fácil trabalhabilidade e de densidade

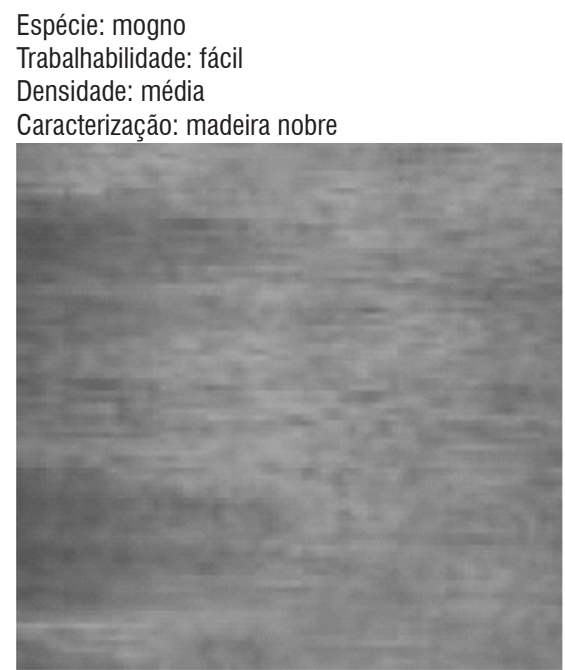

Espécie: cedro
Trabalhabilidade: fácil
Densidade: média
Caracterização: madeira nobre

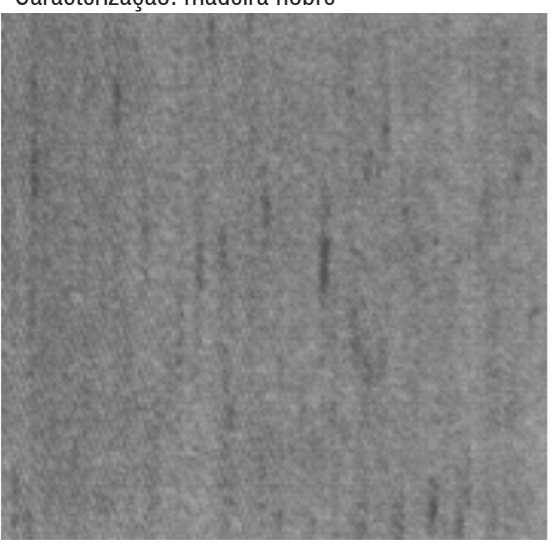

Espécie: louro

Trabalhabilidade: excelente

Densidade: média

Caracterização: madeira vermelha

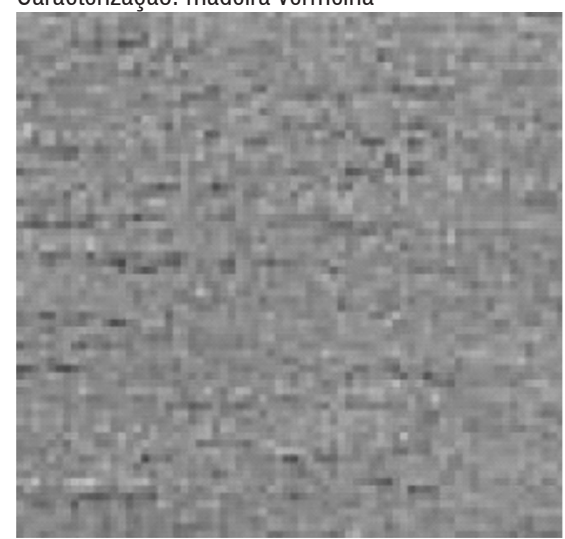

Espécie: angico-branco

Trabalhabilidade: fácil

Densidade: pesada

Caracterização: madeira branca

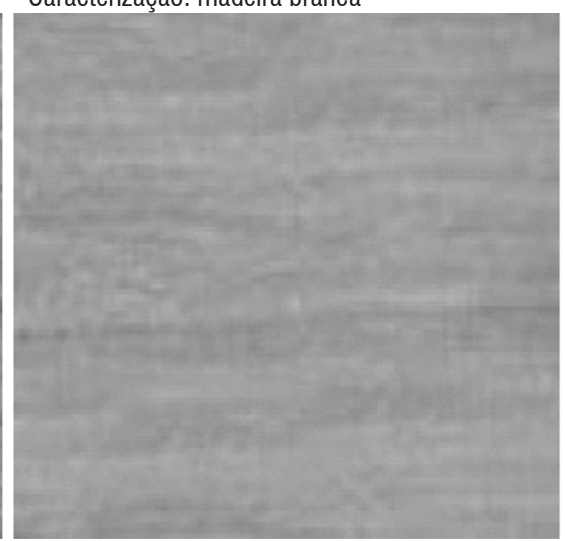

média, identificou-se a espécie como boa substituta ao ipê e à virola.

Essas espécies têm densidades distintas das do mogno: o ipê apresenta maior dificuldade de trabalhabilidade e a virola é caracterizada como uma madeira branca e de menor status no mercado, de baixa densidade, muito utilizada na confecção de compensado (Figura 1).

\section{Fonte: REMADE (2008)}

Nota: Madeira leve (massa específica menor que $500 \mathrm{~kg} / \mathrm{m}^{3}$ ), madeira média (massa específica entre $500 \mathrm{e} 750 \mathrm{~kg} / \mathrm{m}^{3}$ ), madeira pesada (massa específica entre 750 e $950 \mathrm{~kg} /$ $\mathrm{m}^{3}$ ) e madeira muito pesada (massa específica maior que $950 \mathrm{~kg} / \mathrm{m}^{3}$ ). Madeiras nobres são as de maior valor, seguidas, normalmente, pelas madeiras vermelhas e, por último, as brancas. Foras consideradas as fotos e características das espécies de maior ocorrência na Amazônia.

Figura 1 - Algumas características físicas das espécies analisadas.

Provavelmente, o maior número de espécies substitutas do mogno, em relação às outras analisadas, deve-se às dificuldades referentes à garantia de fornecimento da espécie no período analisado. Essas dificuldades estão relacionadas à crescente preocupação com a extinção da espécie, refletida pela sua inclusão no Anexo III da Convenção sobre o Comércio Internacional das Espécies da Flora e da Fauna Selvagens em
Espécie: virola

Trabalhabilidade: fácil

Densidade: leve

Caracterização: madeira branca

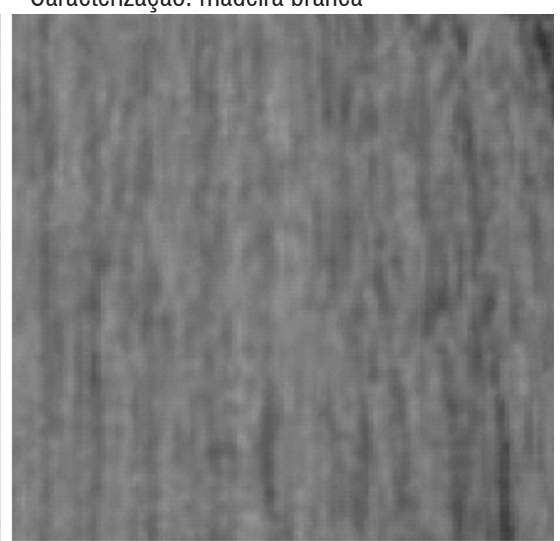

Espécie: ipê

Trabalhabilidade: moderadamente difícil

Densidade: muito pesada

Caracterização: madeira nobre

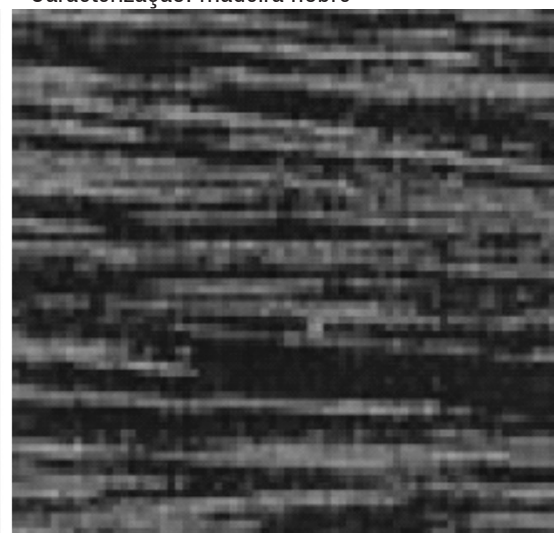

Perigo de Extinção (CITES) desde 1998 e pela inserção no Anexo II em 2002.

Os objetivos da CITES são os de monitorar e deter o comércio internacional das espécies em perigo de extinção. As espécies inclusas no Anexo II requerem um comércio estritamente regulamentado sobre a base de cotas ou 
autorizaçóes que preveem o uso náo-sustentável e dispóem de rigorosos controles de comercialização.

O aumento no controle da comercializaçáo da madeira de mogno levou a uma forte queda em seu volume exportado no período entre 1996 e 2001, anos em que o Brasil exportou mais de 255 mil toneladas de madeira serrada de mogno. Já nos sete anos seguintes (2002 até 2007) esse valor não chegou a 24 mil toneladas (SECEX, 2007), o que, provavelmente, abriu espaço para a entrada de novas espécies no mercado e contribuiu para explicar as elasticidades encontradas.

Ressaltando a substitutibilidade da madeira de mogno por espécies distintas, destaca-se que, apesar da relutância do mercado dos Estados Unidos em se utilizar de madeiras de outras espécies, particularmente no mercado de madeira serrada, há sinais de que a mudança poderia ser iminente (REMADE, 2006). Isso é ilustrado pela troca de espécies populares por espécies alternativas de menor custo. Portanto, a questão-chave a ser trabalhada é quando as espécies menos conhecidas serão viáveis no mercado e não se essas espécies tornar-se-ão viáveis.

Entre as espécies substitutas do mogno, o cedro foi a que apresentou o maior grau de substituição $(-2,2)$ - resultado este de acordo com o esperado, já que ambas as espécies são semelhantes e indicadas, normalmente, para os mesmos fins. Segundo Carvalho (2003), a madeira do cedro é mais mole e de textura mais grossa, porém parecida com a do mogno.

O alto grau de substituição do cedro com o ipê $(-2,4)$ não foi esperado, porém a relação de substituição encontrada, provavelmente, deve-se ao fato de ambas as espécies serem percebidas pelos consumidores como madeiras nobres e semelhantes. Ou seja, apesar de suas diferenças físicas no que tange, principalmente, à trabalhabilidade, densidade e cor, para o mercado, essas espécies são pouco diferenciadas.

Essa hipótese indica que estudos de comportamento do consumidor internacional devem ser realizados a fim de estruturar melhor as estratégias internacionais para espécies brasileiras.

Em relação ao louro, Carvalho (2006) afirma que, em algumas aplicaçóes, ele substitui a madeira de mogno e cedro, quando a cor não é um fator importante. Os resultados confirmaram essa espécie como boa substituta do mogno e sugeriram que, para o mercado internacional, a cor pode ser um fator limitante na sua concorrência com o cedro.

Em relação ao grau de substituição de madeira de louro com a de angico, não foi encontrado na literatura nada a respeito que justificasse o resultado encontrado. Nesse aspecto, destaca-se a facilidade de trabalhabilidade do louro e o seu múltiplo uso, o que o torna um bom substituto para muitas espécies. Segundo Carvalho (2006), além do mogno e cedro, a madeira de louro é um bom substituto da madeira de nogueira e da teca.

\section{CONCLUSÕES}

A análise dos dados e a metodologia utilizada indicaram que:

Há dificuldade para se explicar a substitutibilidade entre as madeiras tropicais no mercado internacional com base apenas em características físicas.

As restriçóes ao corte e ao comércio internacional do mogno permitiram o aparecimento de espécies substitutas.

As madeiras consideradas nobres (mogno, ipê e cedro) apresentaram-se como substitutas entre si.

O grau de substituição entre as espécies deve encontrar explicaçôes nas características dos consumidores dessas madeiras, os quais, não necessariamente, fazem suas decisóes somente pelas características tecnológicas das madeiras.

Pela importância das madeiras tropicais no comércio nacional e internacional, recomenda-se um incremento de pesquisas na área econômica e de mercado, como estudos de comportamento do consumidor, a fim de possibilitar melhorias de estratégias para madeiras brasileiras nos distintos mercados.

\section{BIBLIOGRAFIA CITADA}

Angelo, H. 1998. As exportaçôes brasileiras de madeiras tropicais. Tese de Doutorado, Universidade Federal do Paraná, Curitiba, Paraná. 129 pp.

Ângelo, H. 2006. De motosserras e queimadas. Com Ciência Reportagens. Disponível em: (http://www.comciencia.br/ reportagens/2005/08/04.shtml). Acesso em: 02/12/2006.

Ângelo, H.; Prado, A.C.; Brasil, A.A. 2004. Influência do manejo florestal e do desmatamento na oferta de madeiras tropicais na Amazônia brasileira. Ciência Florestal, 14: 103-110.

Angelo, H.; Silva, D.A. 1998. As exportações brasileiras de mogno (Swetenia macrophylla, King). Revista Árvore, 22: 113-121.

Barreto, P.; Souza Jr., C.; Galvão, C.; Albuquerque, K.; Giselle, A.; Macedo, M.; Firestone, L. 2002. Controle do desmatamento da exploraçấo de madeira na Amazônia: diagnóstico e sugestóes. Relatório Técnico do IMAZON - Versão preliminar para discussão. MMA/PPG7/ProManejo. Belém. 36 pp.

Barreto, P.; Uhl, C. 1993. O potencial de produçâo sustentável de madeira em Paragominas-PA, na Amazônia Oriental: consideraçóes ecológicas e econômicas, p.387-392. In: CONGRESSO FLORESTAL PANAMERICANO, 1 ; CONGRESSO FLORESTAL BRASILEIRO, 7. Anais... SBSSBEF, v.1. Curitiba, Paraná.

Barros, A.C.; Veríssimo, A. 2002. A expansão madeireira na Amazônia: Impactos e perspectivas para o desenvolvimento sustentável no Pará. Belém: Imazon, 180 pp. 
Brandt, S.A. 1980. Comercialização agrícola. Piracicaba, Livroceres. $195 \mathrm{pp}$

Brasil, A.A. 2002. As exportaçóes brasileiras de painéis de madeira. D2002. Dissertação de Mestrado, Setor de Ciências Agrárias/ Universidade Federal do Paraná, Curitiba, Paraná, 74 pp.

Brasil, A.A. 2002. As exportaçōes brasileiras de painéis de madeira. Dissertação de Mestrado, Setor de Ciências Agrárias/Universidade Federal do Paraná., Curitiba, Paraná, 74 pp.

Browder, J.O. 1986. Logging the rainforest: a political economy of timber extraction and unequal exchange in the Brazilian Amazon. Ph.D. Thesis, University of Pennsylvania, Pennsylvania, USA. $335 \mathrm{pp}$.

Calderon, R.; Angelo, H. 2006. As exportaçóes brasileiras de manufaturados de madeira. Ciência Florestal, 16: 99-105.

Calderon, R.A. 2005. Funçöes de oferta e demanda de exportaçóes para manufaturados de madeira. Dissertação de Mestrado, Universidade Federal de Brasília, Brasília, Distrito Federal, 56 pp.

Camargos, J.A.A.; Czarneski, C.M.; Meguerditchian, I.; Iliveira, D. 1996. Catálogo de árvores do Brasil. Brasília, IBAMA/LPF. $888 \mathrm{pp}$.

Carvalho, P.E.R. 2003. Espécies arbóreas brasileiras. Brasília, DF: Embrapa Informaçóes Tecnológicas; Colombo, PR: Embrapa Florestas. 1039 pp.

Carvalho, P.E.R. 2006. Espécies arbóreas brasileiras. Brasília, DF: Embrapa Informaçôes Tecnológicas; Colombo, PR: Embrapa Florestas. 627 pp.

FAO. Monthly Bulletin of Tropical Timber. Rome. (várias ediçôes).

FAO. 1985. Yearbook of forest products 1983. (FAO Forestry Series, 18; FAO Statistical Series, 60), Rome. 408 pp.

FAO. Yearbook of forest products. Rome. (várias edições).

Filha, I.G. 2002. Manejo florestal: questôes econômico-financeiras e ambientais. Estudos Avançados, 16 : 91-1006.

Fontes, R.M.O.; Barbosa, M.L. 1991. Efeitos da integração econômica do Mercosul e da Europa na competitividade das exportações brasileiras de soja. Revista de Economia e Sociologia Rural, 29: 335-351.

Gama, Z.A.G.P.; Braz, E.M. 1993. Identificação do custo de produção do manejo florestal sustentado e seus reflexos na serrarias do estado do Acre, p.363-366. In: CONGRESSO FLORESTAL PANAMERICANO, 1.; CONGRESSO FLORESTAL BRASILEIRO, 7., 1993. Anais... SBS-SBEF, v.1. Curitiba, Paraná.

International Tropical Timber Organization. 2007. Annual review and assessment of the world timber situation. Yokohama, (Document GI-7/07). 53 pp.

IPEA. 2008. Disponível em: (http://www.ipeadata.gov.br/ipeaweb. dll/ipeadata?13 57421). Acesso em: 02/08/ 2008.

Jansen, M.R.A.; Alencar, J.C. 1991. Contribuição à reposição florestal no estado do Amazonas. In: Val, A.L.; Figlioulo, R.; Feldberg, E. (Eds.). Bases científicas para estratégias de preservação e desenvolvimento da Amazônia. Instituto Nacional de Pesquisas da Amazônia - INPA, Vol.1, Manaus, Amazonas.
Lentini, M.; Veríssimo, A.; Sobral, L. Fatos florestais da Amazônia. Belém: Imazon. 110 pp.

Lima, J.E. 2000. Definiçôes alternativas de elasticidade de substituição: revisão e aplicaçáo, Revista de Economia e Sociologia Rural, 38: 9-44.

Medeiros, V.X.; Teixeira, E.C. 1996. Competição no Mercosul e no mercado internacional de carnes. Revista de Economia e Sociologia Rural, 34: 49-70.

Mercado, R.S. 1980. A indústria madeireira na Amazônia: estrutura, produção e mercados. Tese de Doutorado, Michigan State University, Michigan, USA. Tradução: Maria Salete de Teixeira Guedes.

Mercado, R.S.; Campaganini, S. 1988. Exportaçóes da floresta Amazônia, p.43-73. In: ENCONTRO DE ECONOMIA FLORESTAL, 1.Anais... EMBRAPA/Centro Nacional de Pesquisa de Floresta, vol.1, Curitiba, Paraná.

PRÓ EXPORT. 2003. Madeira pode render ao país US\$ 15 bilhôes em dez anos, n.1, 2003. Disponível em: (http://www. moveisdevalor.com.br/proexport/Informativo_Weinig_Proexp. pdf). Acesso em: 16/07/2003.

Queiroz, E.S.P. 1983. Análise da indústria de beneficiamento primário de madeira no estado do Pará. 1983. Dissertação de Mestrado, Universidade Federal do Paraná, Curitiba, Paraná. 92 pp.

REMADE. 2006. Revista da Madeira. Mérito Exportação. Estados Unidos lidera importações brasileiras, n. 96, ano 16. Disponível em: (http://www.remade.com.br/pt/ revista.php?ano=2006). Acesso: 28/08/ 2008.

REMADE. 2008. Revista da Madeira. Disponível em: (http://www. remade.com.br). Acesso: 20/08/2008.

Sabogal, C.; Pokorny, B.; Bernardo, P.; Massih, F.; Boscolo, M.; Lentini, M.; Sobral, L.; Adalberto, V.; Silva, N.; Zweede, J. 2005. Manejo florestal empresarial na Amazônia brasileira: Restriçôes e oportunidades para a adoção de boas práticas de manejo. Belém/PA. 99pp. Disponível em: (http://bommanejo. cpatu.embrapa.br/ arquivos/15.1-Sabogaletal2005.pdf). Acesso em: 25/08/2008.

Santos, J. 1986. Situação da indústria madeireira no município de (1981 a 1983) e das serrarias do Estado do Amazonas (1981). Dissertaçáo de Mestrado, Universidade Federal do Paraná, Curitiba, Paraná. 78 pp.

Santos, J. 1988. Diagnóstico das serrarias e das fábricas de laminados e compensados do estado do amazonas. Acta Amazonica, 18: 67-82.

Santos, J.; Hummel, A.C. 1988. Situação das exportaçôes de madeiras serradas, laminadas e compensadas do Estado do Amazonas (1984, 1985 e 1996), p.415-430. In: ENCONTRO DE ECONOMIA FLORESTAL, 1. Anais... Curitiba, Paraná: EMBRAPA/Centro Nacional de Pesquisa de Floresta, Vol.2.

SECEX. 19Ministério de Comércio Exterior. Relatórios de Exportação. Brasília, 1980/98.

SECEX. 2007. Ministério do Desenvolvimento, Indústria e Comércio Exterior (MDIC), Secretaria de Comércio Exterior (SECEX). Disponível em: (http://aliceweb.desenvolvimento. gov.br/). Acesso: 10/07/2007. 


\section{ACTA}

Silva, D.A.; Frazão, F.J.; Rocha, J.S.; Matos, J.L.M.; Trughilho, P.; Iwakiri, S. 1991. A indústria de base florestal na Amazônia, p.239-250. In: Val, A.L., Figliuolo, R.; Feldberg, E. (Eds.). Bases cientificas para estratégias de preservação e esenvolvimento da Amazônia. Instituto Nacional de Pesquisas da Amazônia - INPA, Vol.1, Manaus, Amazonas.

Silva, J.N.M. 1989. The behaviour of the tropical rain forest Brazilian amazon after logging. Thesis Doctor of Phylosophy, University of Oxford, Oxford. 287 pp.

Terezo, E.F.M. 1999. Status do mogno (Swetenia macrophilla, King) na Amazônia brasileira. Programa Nacional de Florestas - Agenda
Positiva para o Setor Florestal. Disponível em: (www.mma.gov. br). 35 pp.

Veríssimo, A.; Barreto, P.; Tarifa, R.C. 1995. Uhl extraction of a high-value natural resource in Amazônia: the case of Mahogany. Forest Ecology and Management, 72: 39-60.

Vicent, J.R. 1992. The tropical timber trade and sustainable development. Science, 256: 1651-1656.

Recebido em: 02/12/2008

Aceito em: 17/06/2009 
